\title{
In situ texture monitoring for growth of oriented cubic boron nitride films
}

\author{
Dmitri Litvinova) and Roy Clarke \\ Randall Laboratory of Physics, University of Michigan, 500 East University Avenue, Ann Arbor, \\ Michigan 48109-1120
}

(Received 8 October 1998; accepted for publication 14 December 1998)

\begin{abstract}
We report evidence for oriented growth of pure-phase cubic boron nitride on silicon (100) substrates. The films are deposited at high temperatures (up to $1200^{\circ} \mathrm{C}$ ) by reduced-bias ion-assisted sputtering. The growth technique produces highly textured $c$-BN films with relatively large grain size $(\sim 1000 \AA)$ and reduced residual stress as the bias voltage is decreased. We have been able to grow thick (up to $2 \mu \mathrm{m}$ ) cubic boron nitride films containing $100 \%$ of the cubic phase with the (001) crystallographic axis of $c$-BN oriented perpendicular to the surface of the film. We show how reflection high-energy electron diffraction applied to texture monitoring in polycrystalline films can be used as an in situ process control technique that allows texture identification and quantitative characterization of its angular spread. (C) 1999 American Institute of Physics.

[S0003-6951(99)03407-5]
\end{abstract}

It has been a controversal issue whether or not cubic boron nitride $(c$-BN) films exhibit preferential crystallographic orientation and, if so, under what growth conditions can the film texture be improved. Hexagonal boron nitride films grown by ion-assisted deposition develop a strong preferential crystallographic orientation with the $c$ axis lying in the plane of the film. ${ }^{1}$ Since the growth of an oriented buffer layer of hexagonal boron nitride ( $h$ - $\mathrm{BN}$ ) usually preceeds the formation of the cubic phase, the question arises whether or not the $c$-BN layer would align itself with respect to the hexagonal buffer layer in order to minimize the free energy of the system., ${ }^{2,3}$

In this work we show that reduced-bias ion-assisted sputtering can be used to grow highly textured films in the cubic phase of boron nitride. ${ }^{4,5}$ The key to improving the film microstructure is our implementation of recently developed in situ reflection high-energy electron diffraction (RHEED) analysis for texture identification of polycrystalline films. ${ }^{6,7}$ We show that reducing the energy of nitrogen ions used to stabilize the cubic phase leads to a dramatic decrease of surface damage and as a consequence, to a higher degree of texturing.

The growth studies were conducted in a customdesigned ultrahigh vacuum chamber with a base pressure of $10^{-10}$ Torr. $^{8}$ Hot pressed boron nitride of $4 N$ purity was used as a sputtering target and nitrogen ions were supplied from an electron cyclotron resonance (ECR) source. The silicon (100) substrate, which was heated to over $1000^{\circ} \mathrm{C}$ by direct Joule heating, was biased with a negative dc voltage to control the energy of nitrogen ions arriving at the film surface. At this temperature, the low base pressure of our vacuum chamber allowed thermal desorption of oxygen from the substrate surface, eliminating the need for any special surface treatment prior to deposition.

RHEED was used both as an in situ probe of microscopic film structure to check that the cubic rather than hexagonal phase is formed (recognizing that $c$ - BN and $h$-BN

a)Electronic mail: litvinov@umich.edu films have distinct RHEED patterns), and as a technique to characterize the film's texture. ${ }^{6,7}$ We monitored the RHEED pattern using the kSA 400 real-time data aquisition system. ${ }^{9}$ In addition we used ex situ Fourier transform infrared spectroscopy (FTIR) to provide structural information such as $c$-BN content. ${ }^{10,11}$ The vibrational frequencies of the infrared-active phonon modes for the cubic and hexagonal phases of BN are well known and tabulated. ${ }^{12}$ In particular, the $h$ - $\mathrm{BN}$ phase has two modes at $770 \mathrm{~cm}^{-1}\left(A_{2 u}\right.$ mode) and $1383 \mathrm{~cm}^{-1}\left(E_{1 u}\right.$ mode) and $c$-BN has a peak in its absorption spectrum at $\sim 1065 \mathrm{~cm}^{-1}$ corresponding to the TO phonon mode. ${ }^{13}$

The $c$-BN thin film growth was initiated by setting the substrate bias (nitrogen ion energy) at a value that maximizes the nucleation of the cubic phase as determined by FTIR. We found that a bias of $-96 \mathrm{~V}$ produced the largest $s p^{3} / s p^{2}$ ratio $(\sim 90 \%)$ for the deposition conditions selected here (nitrogen current density $1 \mathrm{~mA} / \mathrm{cm}^{2}$, substrate temperature $T$ $\approx 1000^{\circ} \mathrm{C}$, sputtering gun power $500 \mathrm{~W}$ ). The initial phase of the growth (the "template layer") was carried out at this bias for $3 \mathrm{~h}$. Subsequent to the growth of the template layer, the bias voltage was reduced as described below, thus lowering the kinetic energy of the nitrogen ions incident on the surface of the growing film. We refer to this procedure as "reduced bias" growth. ${ }^{4}$

This $3 \mathrm{~h}$ growth period corresponds approximately to a minimum critical thickness of the $c$-BN template layer below which there is no sustained formation of the cubic phase with the reduced nitrogen ion energies, as determined by RHEED and FTIR. The minimum thickness of the BN template layer coincides with the film thickness $(500 \AA)$ at which coalescence of the single crystal $c$-BN grains occurs to form a smooth surface layer. ${ }^{14}$ Thus a growth time of $3 \mathrm{~h}$ is necessary to obtain a film of sufficient thickness to complete the nucleation and coalescence process for the template layer. The growth of the BN template layer was followed immediately by growth at the reduced bias with all other deposition conditions kept fixed. The FTIR absorption spectrum of the template layer on a silicon substrate was used as 


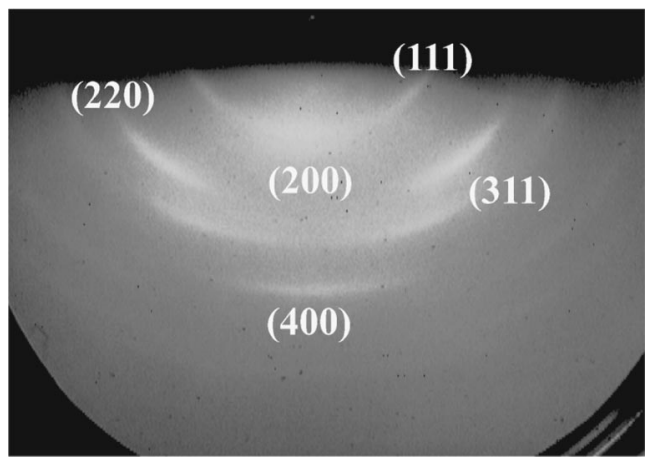

FIG. 1. RHEED patterns for $c$-BN films grown by reduced-bias ion-assisted sputtering.

a background spectrum to obtain FTIR spectra of the films subsequently grown with the reduced substrate bias.

Figure 1 shows a typical RHEED pattern of a cubic boron nitride film grown by reduced-bias ion-assisted sputtering. The presence of diffraction arcs arising from the angular modulation of polycrystalline diffraction rings in the RHEED patterns indicates the textured growth of the films or, in other words, the preferential alignment of the crystallographic axes. ${ }^{6,7}$ We used the approach developed by Litvinov, O'Donnell, and Clarke to identify the texture and to quantitatively characterize its angular dispersion for the films grown by the method described above. First, we notice that the appearance of the RHEED pattern suggests the presence of a fiberlike texture, ${ }^{7}$ i.e., a texture where one of the crystallographic axes is preferentially oriented with respect to the film surface. Also, this crystallographic axis is not parallel to the film surface. We find experimentally that the RHEED patterns from $c$-BN films are invariant to rotation around the film normal which means that the texture axis is aligned along the surface normal ( $z$ axis). After indexing the diffraction rings (cubic boron nitride has zinc-blende crystal structure), we notice that the (002) and (004) diffraction arcs are symmetric around the $z$ axis with the maximum of intensity centered on the $z$ axis. It follows that the (001) crystal axis is the axis with preferential orientation. We conclude that $c$-BN films grown by the deposition technique described above have their (001) crystallographic axis perpendicular to the film surface.

For the cubic crystal lattice, the intensity distribution along the diffraction arcs is described by the following vector ${ }^{7}$

$$
\mathbf{I}(\Phi, \gamma)=A_{0} \frac{d}{\left|\mathbf{k}_{\mathrm{e}}\right|} \frac{2 \pi}{a}\left(\begin{array}{c}
V_{ \pm}(\Phi, \gamma) \\
U_{ \pm}(\Phi, \gamma)
\end{array}\right) D\left(\Delta \Phi_{\|}, \Delta \Phi_{\perp}\right),
$$

where $A_{0}$ is a normalizing constant, $d$ is the perpendicular distance from the sample to the screen, $a$ is a crystal lattice parameter, $\mathbf{k}_{e}$ is the incident electron wave vector, $\Phi$ is the angle between the texture direction and the normal to the film surface ( $z$ axis), and $\gamma$ is the angle between the direction of the incident electron beam and the plane defined by the surface normal and the texture direction ( $j k$ plane). $D\left(\Delta \Phi_{\|}, \Delta \Phi_{\perp}\right)$ is a distribution function that describes angular spread of the texture with quantities $\Delta \Phi_{\|}$and $\Delta \Phi_{\perp}$ being the components of angular spread parallel and perpendicular to the $j k$ plane. The components $V_{ \pm}$and $U_{ \pm}$are defined by ${ }^{7}$

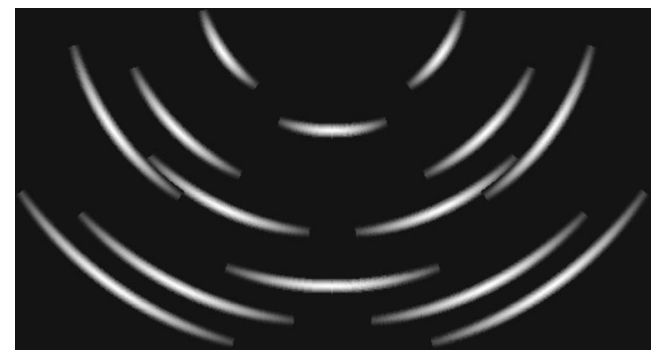

FIG. 2. Simulated RHEED pattern for zinc-blende crystal lattice with (001) texture perpendicular to the plane of the film. Angular spread in texture is $20^{\circ}$.

$$
\begin{aligned}
& V_{ \pm}(\Phi, \gamma)=\frac{-l \cos \gamma \sin \Phi \pm R \cos \Phi}{S^{2}}, \\
& U_{ \pm}(\Phi, \gamma)=\frac{l \cos \Phi \pm R \cos \gamma \sin \Phi}{S^{2}},
\end{aligned}
$$

where $(h k l)$ are the crystallographic indices, $S^{2}=1$ $-\sin ^{2} \gamma \sin ^{2} \Phi$, and $R=\sqrt{-l^{2}+\left(h^{2}+k^{2}+l^{2}\right) S^{2}}$.

The diffraction pattern is then calculated by varying $\Phi$ and $\gamma$ within the range of angles defined by the texture angular spread for each set of $(h k l)$ allowed by the crystal symmetry (zinc-blende structure).

Figure 2 shows a simulated RHEED pattern for the zincblende structure. As one can see there is a close resemblance between the experimental and simulated diffraction patterns.

In the presence of a (001) fiberlike texture perpendicular to the film surface the intensity distribution along the $(00 l)$ diffraction arc is described by the following vector: ${ }^{7}$

$$
\mathbf{I}=A_{0} \frac{d}{\left|\mathbf{k}_{\mathrm{e}}\right|} \frac{2 \pi}{a} l\left(\begin{array}{c} 
\pm \sin \Phi \\
\cos \Phi
\end{array}\right) D\left(\Delta \Phi_{\|}, \Delta \Phi_{\perp}\right) .
$$

It follows that the texture angular spread is equal to the angular width of a $(00 l)$ diffraction arc. This provides an easy way of measuring texture angular spread directly from RHEED patterns.

We analyzed the angular width of the diffraction arcs for a series of films grown under different ion bombardment conditions to determine the angular spread in the texture as a function of ion irradiation energy. Figure 3 shows the dependence of angular spread as a function of substrate bias voltage (nitrogen ion energy). We see that the angular spread in the texture decreases markedly with the decrease of incident nitrogen ion energy, which is consistent with the notion that ion bombardment introduces defects into the film. ${ }^{4}$

Other evidence of improved film quality can be obtained by measuring the radial width of the diffraction arcs, which

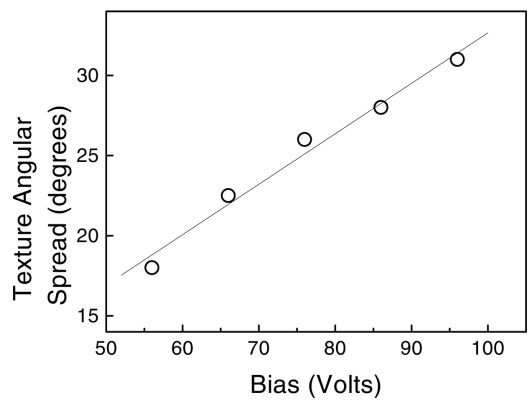

FIG. 3. Angular spread in texture vs substrate bias voltage. 


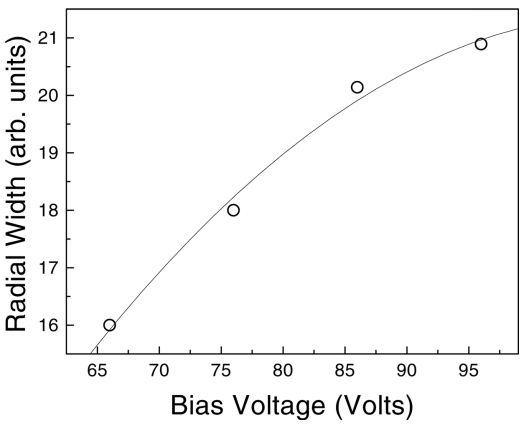

FIG. 4. Radial width (full width at half-maximum) of the (004) diffraction arc vs substrate bias voltage.

is related to the average crystallite size, as a function of the bias voltage. Figure 4 illustrates that the radial width of the (004) diffraction arc decreases as the bias voltage is reduced, again confirming significant improvement in the film microstructure.

In a separate study ${ }^{4}$ of FTIR absorption spectra we have shown that lowering the bias voltage also leads to a decrease of structural defects, which is consistent with our present findings.

Published transmission electron microscopy diffraction patterns for the films grown by mass-separated ion beam deposition ${ }^{15}$ and by reactive dc sputtering ${ }^{16}$ suggest a texture in which at least one (111) crystallographic direction lies in the plane of the film. Ballal and Salamanca-Riba ${ }^{17}$ presented evidence of a strong preferential orientation for the films deposited by ion-assisted pulsed laser deposition having a (110) out-of-plane texture, i.e., the (110) crystallographic direction lines up perpendicular to the plane of the film. Our films grown by ion-assisted rf sputtering exhibit (100) outof-plane texture which is obviously different from the ones mentioned above. Evidently, different deposition techniques result in different preferred crystallographic orientations in the material. Further analysis is necessary to understand which physical processes determine a particular texture in $c$-BN films.

In conclusion, we have presented evidence for oriented growth of pure phase cubic boron nitride on silicon (100) substrates by reduced-bias ion-assisted magnetron sputtering. We have shown that by varying the energy of the incident nitrogen ion beam we can control the microstructure of the film. Texture monitoring based on RHEED is shown to be an effective in situ process control and monitoring technique.

This work is supported by ONR Grant Nos. N00014-91J-1398 and N00014-94-J-0763.

${ }^{1}$ D. R. McKenzie, D. J. H. Cockayne, D. A. Muller, M. Murakawa, S. Miyake, S. Watanabe, and P. Fallon, J. Appl. Phys. 70, 3007 (1991).

${ }^{2}$ D. McKenzie, W. McFall, S. Reisch, B. James, I. Falconer, R. Boswell, H. Persing, A. Perry, and A. Durandet, Surf. Coat. Technol. 78, 255 (1996).

${ }^{3}$ G. Cardinale, D. Medlin, P. Mirkarimi, K. McCarty, and D. Howitt, J. Vac. Sci. Technol. A 15, 196 (1997).

${ }^{4}$ D. Litvinov and R. Clarke, Appl. Phys. Lett. 71, 1969 (1997).

${ }^{5}$ D. Litvinov, C. A. Taylor II, and R. Clarke, Diamond Relat. Mater. 7, 360 (1998).

${ }^{6}$ S. Andrieu and P. Frechard, Surf. Sci. 360, 289 (1996).

${ }^{7}$ D. Litvinov, T. O’Donnell, and R. Clarke, J. Appl. Phys. 85, 2151 (1999).

${ }^{8}$ C. A. Taylor II, Ph.D. thesis, University of Michigan, 1996.

${ }^{9}$ kSA 400 Analytical System, $k$-Space Associates, Inc., Ann Arbor, MI 48104.

${ }^{10}$ D. Kester and R. Messier, J. Appl. Phys. 72, 504 (1992).

${ }^{11}$ S. Reinke, M. Kuhr, W. Kulisch, and R. Kassing, Diamond Relat. Mater. 4, 272 (1995).

${ }^{12}$ D. Hoffman, G. Doll, and P. Eklund, Phys. Rev. B 30, 6051 (1984).

${ }^{13}$ P. Gielisse, S. Mitra, J. N. Plendl, R. Griffis, L. Mansur, R. Marshall, and E. Pascoe, Phys. Rev. 155, 1039 (1967).

${ }^{14}$ C. A. Taylor II and R. Clarke, in Diamond Based Composites, edited by M. Prelas (Kluwer, New York, 1997), pp. 63-113.

${ }^{15}$ H. Hofsäss, C. Ronning, U. Griesmeier, M. Gross, S. Reinke, and M. Kuhr, Appl. Phys. Lett. 67, 46 (1995).

${ }^{16}$ H. Lüthje, K. Bewilogua, S. Daaud, M. Johansson, and L. Hultman, Thin Solid Films 257, 40 (1995).

${ }^{17}$ A. Ballal and L. Salamanca-Riba, Thin Solid Films 224, 46 (1993). 